\title{
Multisensory processing in the redundant-target effect: A behavioral and event-related potential study
}

\author{
MATTHIAS GONDAN \\ University of Regensburg, Regensburg, Germany \\ BIRGIT NIEDERHAUS and FRANK RÖSLER \\ Philipps University, Marburg, Germany \\ and \\ BRIGITTE RÖDER \\ Hamburg University, Hamburg, Germany
}

\begin{abstract}
Participants respond more quickly to two simultaneously presented target stimuli of two different modalities (redundant targets) than would be predicted from their reaction times to the unimodal targets. To examine the neural correlates of this redundant-target effect, event-related potentials (ERPs) were recorded to auditory, visual, and bimodal standard and target stimuli presented at two locations (left and right of central fixation). Bimodal stimuli were combinations of two standards, two targets, or a standard and a target, presented either from the same or from different locations. Responses generally were faster for bimodal stimuli than for unimodal stimuli and were faster for spatially congruent than for spatially incongruent bimodal events. ERPs to spatially congruent and spatially incongruent bimodal stimuli started to differ over the parietal cortex as early as $160 \mathrm{msec}$ after stimulus onset. The present study suggests that hearing and seeing interact at sensory-processing stages by matching spatial information across modalities.
\end{abstract}

In a typical redundant-target experiment, participants are asked to respond to events of two modalities, which are presented either alone or simultaneously (Miller, 1982). Combined (bimodal) stimuli are called redundant because the same reaction is required for both targets, irrespective of their modality. Reaction times (RTs) to redundant targets are commonly found to be shorter than RTs to simple (unimodal) targets. Two alternative models have been suggested to explain this redundant-target effect (RTE). Separate activation models, or race models, hold that the two components of a bimodal event are processed in separate channels and that the channel that has first finished processing first triggers the response. By analogy, the probability of obtaining a six is higher when two dice are tossed instead of one. Similarly, the probability of getting an RT, $t$, lower than a given $t_{0}$ is higher for bimodal (e.g., auditory-visual, AV) than for unimodal (A or V) stimuli, resulting in a lower mean RT. This effect has been called statistical facilitation (Raab, 1962; see Figure 1). Given the RT distributions to simple

\footnotetext{
This study was supported by Grants Ro 1226/4-1 and 4-2 to B.R. from the German Research Foundation (DFG). Correspondence concerning this article should be addressed to M. Gondan, Institute for Experimental Psychology, University of Regensburg, Universitätsstr. 31, D-93050 Regensburg, Germany (e-mail: matthias.gondan@psychologie. uni-regensburg.de).
}

stimuli, the redundancy gain that can be explained by statistical facilitation has a clearly defined upper limit, which is described by the race model inequality (Miller, 1982):

$$
p\left(t<t_{0} \mid \mathrm{AV}\right) \leq p\left(t<t_{0} \mid \mathrm{A}\right)+p\left(t<t_{0} \mid \mathrm{V}\right) .
$$

A race model requires that this inequality should hold for the cumulative RT distributions for both unimodal and bimodal stimuli. If the redundancy gain surpasses that predicted by the race model, rejecting the race model in favor of a coactivation model is justified. Proponents of the latter model disagree with the separate processing view and suggest that information from the two modality channels is integrated at a particular processing level and subsequently processed as a combined entity. This processing stage gains from redundant information, resulting in faster responses to redundant stimuli.

The race model is typically sufficient to explain the redundancy gain of healthy participants in simple unimodal detection tasks with two classes of visual stimuli (see, e.g., Corballis, 1998, 2002; a weak violation was found by Miniussi, Girelli, \& Marzi, 1998, which was, however, not statistically tested). Surprisingly, split-brain patients have displayed redundancy gains larger than those predicted by the race model when bilateral stimuli have been used (Corballis, 1998, 2002; Reuter-Lorenz, Nozawa, Gazzaniga, \& Hughes, 1995; Roser \& Corballis, 2002). 

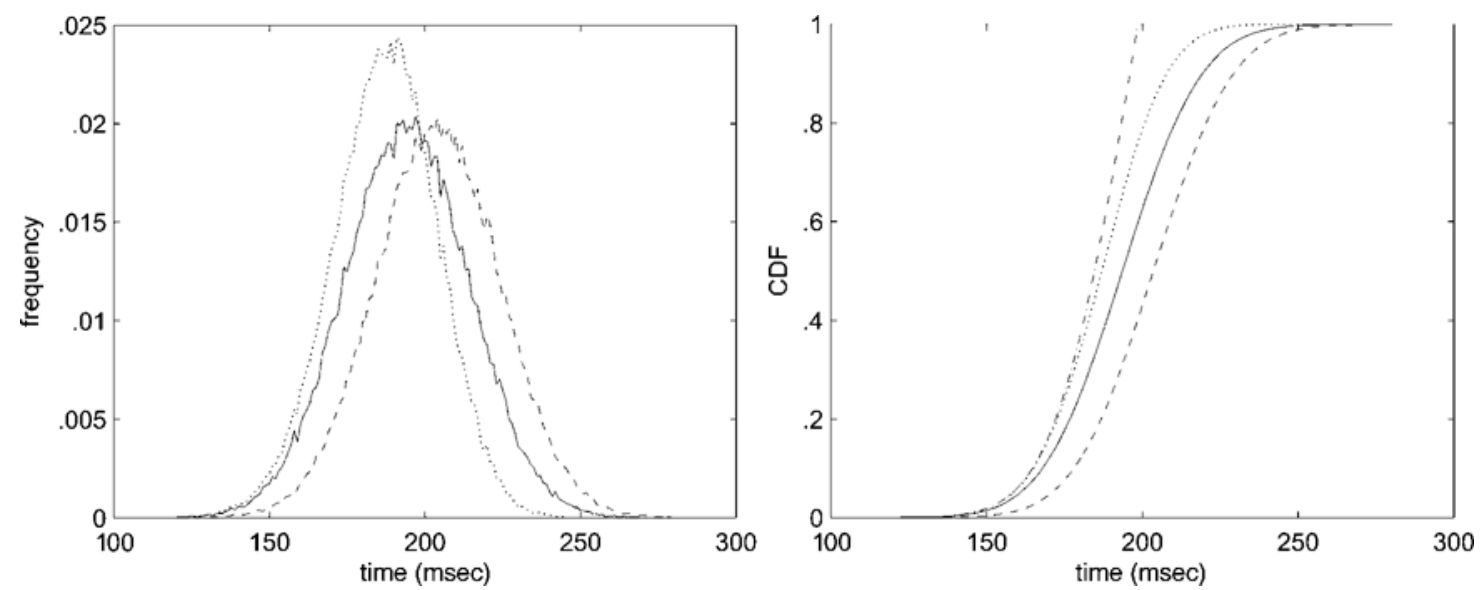

Figure 1. A hypothetical example for statistical facilitation. The solid and dashed lines indicate reaction time (RT) distributions of stimuli from two different modalities; the dotted line indicates the RT distribution for redundant stimuli in a perfect race model. On the right, the corresponding cumulative distribution probability functions (CDFs) are plotted. The dotted-dashed line shows the sum of the simple distributions. Note that in the cumulative distribution on the right, the dotted line never crosses the summed simple RT distribution (race model inequality; see Equation 1).

Therefore, Roser and Corballis concluded that coactivation occurs at the subcortical, rather than at the cortical, level. By contrast, fast interhemispheric transfer might mask subcortical coactivation in healthy individuals.

For bimodal divided attention tasks, the RT gain observed with bimodal stimuli is usually larger than that predicted by separate activation models, so that coactivation models have been adopted (Giray \& Ulrich, 1993; Gondan, Lange, Rösler, \& Röder, 2004; Hughes, ReuterLorenz, Nozawa, \& Fendrich, 1994; Miller, 1982, 1986, 1991; Molholm et al., 2002; Plat, Praamstra, \& Horstink, 2000; Schröger \& Widmann, 1998). Different loci at which the coactivation may take place have been suggested: Multisensory interactions may take place (1) at perceptual stages (Hershenson, 1962; Hughes et al., 1994; Molholm et al., 2002), (2) at higher cognitive stages (e.g., decision or memory; Miller, 1982; Mordkoff \& Yantis, 1991; Schröger \& Widmann, 1998), and/or (3) during motor preparation and execution (Diederich \& Colonius, 1987; Giray \& Ulrich, 1993; but see Miller, Ulrich, \& Lamarre, 2001; Mordkoff, Miller, \& Roch, 1996).

With event-related potentials (ERPs), multisensory interactions have typically been investigated using unimodal stimuli of two modalities and their bimodal combination (e.g., auditory/A, visual/V, and bimodal/AV). Unimodal stimuli evoke typical sensory-specific potentials. The two unimodal ERPs are summed and subtracted from the ERPs to bimodal stimuli: AV - $(\mathrm{A}+\mathrm{V})$. A nonzero result is interpreted as an interaction of the two modalities. Using this formula, Foxe et al. (2000) demonstrated an interaction of the auditory and the somatosensory system at right-central recording sites, for stimuli presented from the left side, as early as $60 \mathrm{msec}$ after stimulus onset. These findings received partial support from Lütkenhöner, Lammertmann, Simões, and Hari (2002), who measured event-related magnetic fields (ERFs) to uni- and bimodal auditory and tactile stimuli: The bimodal ERF differed from the summed unimodal ERFs over somatosensory brain regions, starting at $140 \mathrm{msec}$ poststimulus. The authors argued that the timing and the stimuli in the two studies differed considerably, which may be the reason for the diverging results.

In a recent study by Molholm et al. (2002), redundancy gains were investigated with auditory, visual, and bimodal target stimuli. The RTs were considerably lower for bimodal stimuli than for unimodal stimuli and violated the predictions of the race model. The redundancy gain was accompanied by bimodal ERPs over parietooccipital recording sites that, starting at $56 \mathrm{msec}$, could not be explained by the sum of the unimodal ERPs. Later ERP effects emerged over fronto-central sites at around $120 \mathrm{msec}$ and over central electrodes at $180 \mathrm{msec}$ after stimulus presentation. The timing and the pattern of these ERP effects resembled the findings of Giard and Peronnet (1999), who had used a more complex auditoryvisual classification task. Giard and Peronnet reported evidence for a multisensory interaction over occipital regions, starting at around $40 \mathrm{msec}$ after stimulus onset and over the central cortex, starting around $180 \mathrm{msec}$. Correspondingly, Fort, Delpuech, Pernier, and Giard (2002) have reported ERP effects pointing toward multisensory interactions at a latency of $170 \mathrm{msec}$ after stimulus onset, although only with a subgroup of participants who had lower RTs to auditory than to visual targets. They used a nonredundant target identification task, with target stimuli defined by auditory, visual, and two types of bimodal features - an easy condition $\left(\mathrm{AV}_{1}\right)$ and a difficult condition $\left(A V_{2}\right)$. Since the ERPs to $A V_{1}$ and $A V_{2}$ did not differ until $500 \mathrm{msec}$ after stimulus onset, the authors concluded that the earlier multisensory interaction took 
place at perceptual-processing stages, unaffected by the difficulty of the task. Moreover, Schröger and Widmann (1998) provided electrophysiological and behavioral evidence for redundancy gains in a bimodal go/no-go task: Audiovisual stimuli were presented at a standard position on the left side (S), while participants had to react to three types of deviants, with the auditory (A), the visual $(\mathrm{V})$, or both (AV) stimulus components being presented on the right side. Importantly, all the stimuli were bimodal; only the positions of the auditory and the visual stimulus components were varied. Target minus standard ERP difference waves were calculated separately for each deviant: A - S, V - S, and AV - S. From $190 \mathrm{msec}$ on, the summed difference waves for simple deviants differed from the bimodal difference wave at all electrode sites: $(\mathrm{AV}-\mathrm{S}) \neq(\mathrm{A}-\mathrm{S})+(\mathrm{V}-\mathrm{S})$. It may be concluded that with this type of target detection task, coactivation takes place at a later, nonperceptual stage of processing.

In the $\mathrm{AV}-(\mathrm{A}+\mathrm{V})$ comparison term used in all the studies cited, the two unimodal ERPs are subtracted from one bimodal ERP. In the study by Schröger and Widmann (1998), the same procedure was applied to the difference waves. As Teder-Sälejärvi, McDonald, di Russo, and Hillyard (2002) pointed out, this comparison is inappropriate if there is common activity (C) in the three ERPs (such as, e.g., target expectation, target detection, etc.), because the common activity is subtracted twice from the bimodal ERP. The resulting term reflects not only multisensory interactions, but also the inverse of the common activity: AVC $-(\mathrm{AC}+\mathrm{VC})=-\mathrm{C}$. The present study suggests an alternative comparison. Two bimodal ERPs were subtracted from two other ERPs, with the spatial relationship of the two components of the bimodal stimulus being manipulated. If two ERPs are subtracted from two others, the common activity $\mathrm{C}$ is eliminated from the resulting term.

Animal studies have uncovered several brain structures with multisensory responsiveness-for example, the superior colliculus (SC; see Stein \& Meredith, 1993), regions in the posterior parietal cortex (Carlson, Pertovaara, \& Tanila, 1987; Mazzoni, Bracewell, Barash, \& Andersen, 1996), and the anterior ectosylvian sulcus in the cat (Wallace, Meredith, \& Stein, 1992). Multisensory neurons in the deeper layers of the SC (from Layer II on) have spatially overlapping receptive fields for visual, auditory, and somatosensory inputs (Meredith \& Stein, 1987). Although these neurons react to unimodal input from either modality, bimodal stimuli lead to a strong response enhancement: Firing rates to bimodal stimuli exceed by far the summed firing rates to unimodal stimuli, provided that the two stimulus components are presented from the same location. According to Stein and Meredith (1993), this mechanism is important for orienting behavior quickly - in particular, if stimuli in the external world are of low intensity. Consequently, Harrington and Peck (1998) could demonstrate coactivation in saccadic
RTs to spatially congruent auditory-visual target stimuli. With increasing distance between the auditory and the visual target stimuli, the amount of coactivation decreased and was virtually absent for distances over $20^{\circ}$.

In the present experiment, a target detection task with unimodal auditory, unimodal visual, and bimodal stimuli was used. Standards ( $70 \%$, no response needed) were simple light flashes or noise bursts; targets were rapidly repeated light flashes and noise bursts and required a response. They were presented from two loudspeakers with embedded light-emitting diodes (LEDs), and the spatial relationship of the two components of the bimodal stimuli was manipulated: Both components were presented either from one loudspeaker (spatially congruent stimuli) or from opposite locations-for example, the auditory component from the left and the visual component from the right side (spatially incongruent stimuli). The second manipulation was the response relevance of the stimulus components: A bimodal stimulus was either (1) a combination of two standards (and therefore, no response was required), (2) a standard and a target that required a response, or (3) the combination of two targets - namely, the redundant targets.

First, we expected an RT gain for redundant targets, as compared with unimodal simple targets. Second, we tested whether an additional stimulus without response relevance would produce the same RT gain as an additional target stimulus (Mordkoff et al., 1996; Nickerson, 1973). Third, we examined whether the spatial relationship between the two stimuli of a bimodal event would influence the RTs and the size of the RTE. For this purpose, RTs to spatially congruent bimodal stimuli were compared with those to spatially incongruent bimodal stimuli.

ERPs were recorded in order to determine the timing and localization of multisensory interactions, defined as the following: ERPs to spatially congruent auditory-visual stimuli, presented from the same side, were summed and compared with the summed ERPs to spatially incongruent stimuli, presented from opposite sides. Since this comparison contains two minuends (AV left, $\mathrm{AV}$ right) and two subtrahends (A left and V right, A right and $\mathrm{V}$ left), the problem of subtracting one ERP from two is avoided, although the physical stimulation is the same for spatially congruent and spatially incongruent stimuli. The time point at which this difference deviates from zero indicates the processing stage (early perceptual or later target-detection-related processes) at which the spatial representations of both modalities interact.

\section{METHOD}

\section{Participants}

Twelve right-handed students (mean age, 24.5 years; range, 20-31 years) participated. All were free of neurological disorders and had normal hearing and normal or corrected-to-normal vision (self-report). They received course credit or payment and gave written informed consent. The experiment was performed in accor- 
dance with the ethical standards laid down in the 1964 Declaration of Helsinki. EEG data from 3 other participants had to be discarded due to a low signal-to-noise ratio in the ERP data.

\section{Stimuli}

The experiment followed a typical oddball design with frequent $(70 \%$, standards $)$ and rare $(30 \%$, targets $)$ stimuli. Unimodal auditory and visual and bimodal audiovisual stimuli were presented from two positions (left and right). The auditory standards were bursts of white noise of 200-msec duration $(\mathrm{N})$, emitted by one of two loudspeakers located $20^{\circ}$ to the left and to the right of the participant's straight-ahead line, at a distance of $1 \mathrm{~m}$. The auditory target stimuli were double bursts $(\mathrm{NN})$ of $70-\mathrm{msec}$ duration, interrupted by $70 \mathrm{msec}$ of silence. A 200-msec LED flash (L) was used as the visual standard. The LEDs were mounted into the casing of each loudspeaker and were visible through the front grid (four LEDs per loudspeaker). Correspondingly, visual targets were double LED flashes (LL), with the same timing as the auditory targets. In total, eight different standard stimuli were delivered (S1-S8; see Table 1, left column): four unimodal types ( $\mathrm{N}$ left, $\mathrm{N}$ right, $\mathrm{L}$ left, and $\mathrm{L}$ right) and four different bimodal types. The bimodal stimuli were presented either from the same location (spatially congruent stimuli, NL left and NL right) or from different locations (spatially incongruent stimuli, $\mathrm{N}$ left and $\mathrm{L}$ right or $\mathrm{L}$ left and $\mathrm{N}$ right). No response was required for these stimuli.

There were eight different target conditions (T1-T8; middle column of Table 1): four unimodal types (NN left, NN right, LL left, and LL right) and four bimodal types - namely, the redundant targets. Correspondingly, the bimodal targets were presented either at the same location (spatially congruent stimuli: NNLL left and NNLL right) or from different locations (spatially incongruent stimuli: NN left and LL right or NN right and LL left).

A last group of stimuli consisted of bimodal combinations of standards and targets $(\mathrm{C} 1-\mathrm{C} 8$; right column of Table 1$)$ : four conditions in which a noise target was accompanied by a light standard (spatially congruent, NNL left and NNL right; spatially incongruent, $\mathrm{NN}$ left and $\mathrm{L}$ right or $\mathrm{NN}$ right and $\mathrm{L}$ left) and four conditions in which a light target was accompanied by a noise standard (spatially congruent or incongruent, respectively).

The participants were instructed to fixate a cross straight ahead at a distance of $1 \mathrm{~m}$ and to respond to any auditory or visual target (i.e., a double noise or a double light, alone or in combination with a standard, or a double noise and a double light) by releasing a foot pedal placed under the right foot as quickly as possible. In addition, they were told that the redundant targets required only one (not two) responses. The interstimulus interval varied between 1,800 and $2,300 \mathrm{msec}$. After a practice run, 10 blocks of about 12-min duration each were presented. Each standard stimulus (S1-S8) occurred 430 times during the experiment (43 in each of the 10 blocks), and each target occurred 80 times (T1-T8 and C1-C8), yielding a total of 5,520 trials. The experiment took place in a dimly lit, electrically shielded room.

\section{EEG Recording}

The EEG was recorded from 124 equally distantly located scalp electrodes (nonpolarizable $\mathrm{Ag} / \mathrm{AgCl}$ electrodes) mounted into an elastic cap (Easy Cap, FMS). The left mastoid served as the reference. The electrode impedance was kept at $5 \mathrm{k} \Omega$ or below by preparing the skin with "Every" (gelimed) and isopropyl alcohol. Eci Electrogel (Electrocap International) was used as an electrolyte for all the recordings. The band-pass of the amplifiers (Neuroscan) was set from 0.1 to $100 \mathrm{~Hz}$; the sampling rate was $500 \mathrm{~Hz}$. Horizontal eye movements were monitored with a bipolar recording of two electrodes attached to the outer canthi of the eyes (EOG channels). Vertical eye movements were measured with an electrode placed under the left eye, against the reference. The high number of EEG channels served for the evaluation of current density reconstruction algorithms, which will not be reported here. Instead, 17 electrode triplets, as shown in Figure 2, were used for the ERP analyses.

\section{Behavioral Data}

Redundant-target effects were assessed by separately analyzing RTs and omissions to the unimodal target stimuli (T1-T4; see Table 1) and their bimodal combinations (T5-T8) as dependent variables. In addition, false alarms to standard stimuli were recorded. Mean RTs to the targets were calculated for each participant and deviant condition and were submitted to a repeated measures analysis of variance (ANOVA) with the 16 conditions (T1-T8 and C1-C8) as a within-subjects factor. To test the hypotheses put forward in the introduction, the following contrasts were defined: In the first contrast, the four unimodal targets (T1-T4) were compared with all bimodal targets (T5-T8 and $\mathrm{C} 1-\mathrm{C} 8$ ); in a second, standard-target

Table 1

Stimuli Used in the Experiment

\begin{tabular}{|c|c|c|c|c|c|c|c|c|}
\hline \multicolumn{3}{|c|}{$\begin{array}{c}\text { Standards } \\
\text { (No Response Required) }\end{array}$} & \multicolumn{3}{|c|}{$\begin{array}{c}\text { Targets } \\
\text { (Response Required) }\end{array}$} & \multicolumn{3}{|c|}{$\begin{array}{c}\text { Combinations } \\
\text { (Response Required) }\end{array}$} \\
\hline Label & Left & Right & Label & Left & Right & Label & Left & Right \\
\hline S1 & $\mathrm{N}$ & & T1 & NN & & C1 & NN & $\mathrm{L}$ \\
\hline S2 & & $\mathrm{N}$ & T2 & & $\mathrm{NN}$ & C2 & $\mathrm{L}$ & $\mathrm{NN}$ \\
\hline S3 & L & & T3 & LL & & C3 & LL & $\mathrm{N}$ \\
\hline S4 & & $\mathrm{L}$ & T4 & & LL & C4 & $\mathrm{N}$ & LL \\
\hline S5 & $\mathrm{N}$ & $\mathrm{L}$ & T5 & $\mathrm{NN}$ & LL & C5 & NNL & \\
\hline S6 & $\mathrm{L}$ & $\mathrm{N}$ & T6 & LL & NN & C6 & & NNL \\
\hline S7 & NL & & T7 & NNLL & & C7 & LLN & \\
\hline S8 & & NL & T8 & & NNLL & C8 & & LLN \\
\hline
\end{tabular}

Note-Eight different standard stimuli (left column) were used in the experiment, unimodal auditory (S1 and S2) and visual (S3 and S4) stimuli and bimodal stimuli, either from opposite locations (S5 and S6, spatially incongruent displays) or from the same side (S7 and S8, spatially congruent). The corresponding target stimuli are shown in the middle column. Targets were again unimodal (T1-T4) or bimodal - that is, redundant (T5-T8). Like the standards, the bimodal targets were either spatially incongruent (T5 and T6) or spatially congruent (T7 and T8). In addition, combinations of standards and targets, as shown in the right column $(\mathrm{C} 1-\mathrm{C} 8)$, were employed, again either spatially incongruent $(\mathrm{C} 1-\mathrm{C} 4)$ or congruent $(\mathrm{C} 5-\mathrm{C} 8)$. N, simple noise burst; L, simple light flash; NN, double noise burst (target); LL, double light flash (target). 


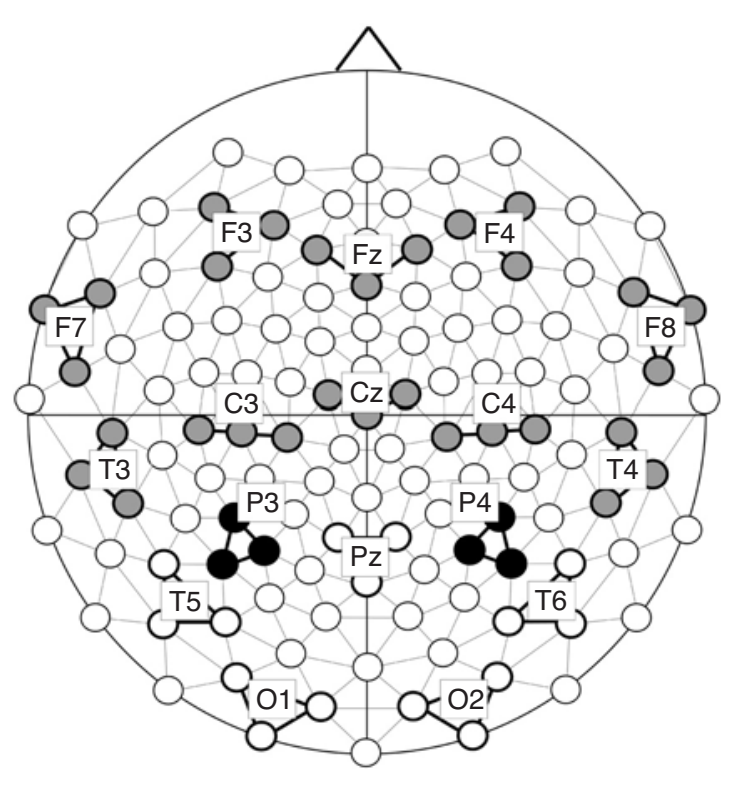

Figure 2. Electrode clustering used for the ERP analysis. Three a priori contrasts were defined for the analysis of the topography of spatial congruency effects: (1) a global front-to-back comparison (gray vs. black/white), (2) parietal recording sites versus surroundings (black vs. white), and (3) P3 versus P4.

combinations $(\mathrm{C} 1-\mathrm{C} 8)$ were compared with the redundant targets (T5-T8); in a third, the spatially congruent and the spatially incongruent standard-target combinations $(\mathrm{C} 1-\mathrm{C} 4$ vs. $\mathrm{C} 5-\mathrm{C} 8)$ were compared; and in a fourth, the spatially congruent and the spatially incongruent redundant targets (T5 and T6 vs. T7 and T8) were compared.

Cumulative distribution functions (CDFs) of the RTs to auditory, visual, and bimodal stimuli were calculated as described in Corballis (2002). For each participant, 18 RT bins of equal size were formed, using the entire data set. In a second step, the number of RTs to auditory, visual, and bimodal stimuli that were faster than a given bin was counted and divided by the total number of correct responses. Finally, a binomial test was run for each bin $(b)$, to test whether $p\left(\operatorname{bin}_{b} \mid \mathrm{AV}\right)$ was larger than $p\left(\operatorname{bin}_{b} \mid \mathrm{A}\right)+p\left(\operatorname{bin}_{b} \mid \mathrm{V}\right)$, using the data for the 12 participants. This test was run for the CDF of each redundant target and the CDFs of the respective unimodal components; for example, the CDF for Condition T5 (NN left and LL right) was compared with the CDF for $\mathrm{T} 1$ (NN left) and the CDF for T4 (LL right). A coactivation effect in this test would indicate a redundancy gain somewhere in the processing pathway; hence, there would be coactivation (1) because of more stimulus energy in the bimodal stimuli (Bernstein, Clark, \& Edelstein, 1969; Nickerson, 1973), (2) due to the fact that both target stimuli activate response codes (Cohen \& Shoup, 1997), or (3) due to more efficient multisensory integration in spatially congruent stimuli (this effect should be visible only in $\mathrm{T} 7$ and $\mathrm{T} 8$ ).

A second race model test was conducted using only bimodal stimuli with a different number of targets, therefore comparing the RT distributions for the redundant targets (e.g., NNLL left) and the two respective standard-target-combinations (NNL left and LLN left). In this test, only the response relevance of the stimuli is manipulated, whereas the number of stimuli and the spatial relationship is kept constant. A coactivation effect in this race model test could be interpreted as evidence for (2), although a contribution of response competition in the combinations of standards and targets (NNL/LLN) cannot entirely be ruled out.

\section{ERP Data}

The EOG channels served for off-line rejection of trials with excessive eye artifacts. Segments with EOG activity larger than $50 \mu \mathrm{V}$ within the epoch between $100 \mathrm{msec}$ before and $400 \mathrm{msec}$ after stimulus onset were rejected. ERPs to each condition were averaged separately, baseline corrected to the mean activity occurring from 50 to $0 \mathrm{msec}$ preceding stimulus onset, and rereferenced off line to the mean voltage of both mastoids.

To investigate multisensory interactions, the two ERPs elicited by spatially congruent bimodal standard stimuli $(\mathrm{S} 7+\mathrm{S} 8, \mathrm{NL}$ left + NL right; see Table 1) were summed and compared with the sum of the two ERPs elicited by spatially incongruent stimuli (S5 + $\mathrm{S} 6, \mathrm{~N}$ left and L right $+\mathrm{L}$ left and $\mathrm{N}$ right). As in the $\mathrm{AV}-(\mathrm{A}+$ $\mathrm{V})$ comparison, any physical stimulus energy is eliminated, and only the effect due to spatial congruency remains (Table 2B). Since left- and right-sided stimuli have to be pooled in this comparison, the laterality information is lost. The mean amplitudes of ERPs to the bimodal standards (S5-S8) were submitted to a $2 \times 2$ repeated measures ANOVA with factors of noise position and light position (see Tables 2A and 2B). In this analysis, the spatial congruency effect would be reflected by an interaction of noise position and light position; that is, the analysis tests whether $(\mathrm{S} 7+\mathrm{S} 8)-(\mathrm{S} 5+\mathrm{S} 6)$ is different from zero. A significant effect was considered reliable if it was observed in five or more consecutive sampling points $(10 \mathrm{msec})$. Huynh and Feldt's (1976) procedure was used to correct the degrees of freedom in the ANOVAs. The results were compared with the respective qualitative findings for target stimuli $[(\mathrm{T} 7+\mathrm{T} 8)-$ $(\mathrm{T} 5+\mathrm{T} 6)]$, combinations of noise targets and light standards $[(\mathrm{C} 7+\mathrm{C} 8)-(\mathrm{C} 3+\mathrm{C} 4)]$, and combinations of noise standards and light targets $[(\mathrm{C} 5+\mathrm{C} 6)-(\mathrm{C} 1+\mathrm{C} 2)]$.

To examine the laterality of the spatial congruency effect, the comparison method above was modified by replacing a bimodal stimulus in the minuend and one in the subtrahend by one of its unimodal stimulus components (see Tables $3 \mathrm{~A}$ and $3 \mathrm{~B}$ ). For example,

Table 2A

ANOVA Model for Testing the Effects of Noise and Light Positions and of Spatial Congruency for Bimodal Standards (Table 1, Left Column) on ERPs

\begin{tabular}{ccc}
\hline & \multicolumn{2}{c}{ Noise Position } \\
\cline { 2 - 3 } Light Position & Left & Right \\
\hline Left & S7 & S6 \\
Right & S5 & S8 \\
\hline
\end{tabular}

Note-The main effects of noise position and light position reflect the laterality of the auditory and visual stimulus components of the bimodal stimuli. In the noise $\times$ light interaction, $(\mathrm{S} 7+\mathrm{S} 8)$ is compared with $(\mathrm{S} 5+\mathrm{S} 6)$. Table 2B shows this interaction in more detail.

Table 2B

Stimuli for the Interaction in the ANOVA in Table 2A

\begin{tabular}{cll}
\hline Stimulus Type & Left & Right \\
\hline S7 & NL & \\
S8 & & NL \\
S5 & N & L \\
S6 & L & N \\
\hline
\end{tabular}

Note-For the interaction in the ANOVA shown in Table 2A, the two ERPs to spatially congruent bimodal stimuli-S7 (noise and light from the left side) and S8 (noise and light from the right side) - are summed and compared with the sum of the two ERPs to spatially incongruent stimuli (S5 and S6). Thus, in (S7 + S8) - (S5 + S6), the physical stimulation is completely eliminated, and the influence of spatial congruency is isolated. 
Table 3A

ANOVA Models for Testing the Laterality of Spatial Congruency Effects on the ERPs, Using Unimodal Light and Bimodal Stimuli

\begin{tabular}{cccccc}
\hline \multirow{2}{*}{ Light Position } & \multicolumn{2}{c}{ Noise Present (Left) } & & \multicolumn{2}{c}{ Noise Present (Right) } \\
\cline { 2 - 3 } \cline { 5 - 6 } & Left & Absent & & Absent & Right \\
\hline Left & S7 & S3 & & S3 & S6 \\
Right & S5 & S4 & & S4 & S8 \\
\hline
\end{tabular}

Note-The main effect of noise present (left) reflects the influence of a noise stimulus on the left side. Light position reflects the laterality of the visual stimulus. In the noise $\times$ light interaction, $(\mathrm{S} 7+\mathrm{S} 4)$ is compared with $(\mathrm{S} 5+\mathrm{S} 3)$. Correspondingly, the right ANOVA tests for the effect of a noise stimulus on the right side. Table 3B shows the interactions of the two ANOVAs in more detail.

S4 (L right) was used instead of S8 (NL right) in the minuend, and S3 (L left) was used instead of S6 (L left/N right) in the subtrahend. The main effect of noise position (left or right) changes thereby to one of noise presence (left or absent); the interaction [(S7 + S4) $(\mathrm{S} 5+\mathrm{S} 3)]$ reflects the influence of a spatially congruent stimulus on the left side.

An analogous comparison was performed with noise presence (right or absent), thus evaluating $(\mathrm{S} 3+\mathrm{S} 8)-(\mathrm{S} 4+\mathrm{S} 6)$, with light left or absent $(\mathrm{S} 7+\mathrm{S} 2)-(\mathrm{S} 1+\mathrm{S} 6)$, and light left or absent $(\mathrm{S} 1+$ $\mathrm{S} 8)-(\mathrm{S} 5+\mathrm{S} 2)$.

To examine topographical differences between the conditions, a third factor, channel, was included in the analysis, representing the 17 electrode triplets. For this variable, three main contrasts were defined: The activity of (1) frontal channels was compared with that of posterior channels, (2) parietal channels (P3 and P4) were contrasted with the surrounding channels, and (3) the laterality was tested by comparing P3 and P4 (see Figure 2).

\section{RESULTS}

\section{Behavioral Data}

False alarms to standard stimuli were, on average, below $2 \%$ and were, therefore, not further analyzed. RTs and omission rates for all the deviant conditions are shown in Table 4: Redundant targets were better detected (i.e., fewer omissions) than single targets $[F(1,11)=$ $25.19, p<.001]$. An overall ANOVA of the RTs in all 16 conditions was highly significant $[F(15,165)=26.98$, corrected $p<.001$, eps $\left._{\mathrm{HF}}=0.17\right]$. The contrast analysis revealed significant effects for all four planned comparisons: The participants reacted more quickly to bimodal target stimuli (T5-T8 and $\mathrm{C} 1-\mathrm{C} 8$; see Table 1) than to unimodal targets (T1-T4), with a mean difference of $38 \mathrm{msec}(S E=11 \mathrm{msec} ; F(1,11)=156.78$, one-tailed $p<$ $.001]$. Within the bimodal stimuli, responses to redundant targets (T5-T8) were faster than those to standard-target combinations $(\mathrm{C} 1-\mathrm{C} 8)$, with a mean difference of $52 \mathrm{msec}$ $[S E=11 \mathrm{msec} ; F(1,11)=265.60$, one-tailed $p<.001]$. Finally, responses were faster for the spatially congruent bimodal stimuli than for the spatially incongruent bimodal stimuli. This effect was observed both in the standardtarget combinations $[\mathrm{C} 1-\mathrm{C} 4$ vs. $\mathrm{C} 5-\mathrm{C} 8$, mean difference $=5.1 \mathrm{msec}, S E=5.7 \mathrm{msec} ; \mathrm{F}(1,11)=9.51$, onetailed $p<.01]$ and in the redundant targets [T5 and T6 vs. $\mathrm{T} 7$ and $\mathrm{T} 8$, mean difference $=4.7 \mathrm{msec}, S E=$ $8.1 \mathrm{msec} ; \mathrm{F}(1,11)=4.18$, one-tailed $p<.05]$.

The race model inequality was violated in all four redundant-target conditions (see Figure 3), when the CDFs for the unimodal targets were summed and compared with the CDFs for the bimodal targets. Binomial tests, as suggested by Corballis (2002), were significant from the first to the eighth RT bin, thus indicating coactivation for all the redundant targets, in both the spatially congruent and the spatially incongruent displays.

In the second race model test, redundant targets were compared with bimodal combinations of standards and targets with the same spatial relationship. The race model violation was generally lower, but still significant (see Figure 4).

\section{ERP Data: Spatial Congruency Effect}

To investigate the multisensory interaction of auditory and visual stimuli, the ERP amplitudes to bimodal standard stimuli (S5 ...S8) were subjected to a three-way repeated measures ANOVA with the factors of noise position, light position, and channel. The results are shown in Table 5 for mean amplitudes of 10-msec time epochs. The noise position $\times$ channel interaction was significant

Table 3B

Spatial Congruency Effect as Reflected by the

Interactions in the ANOVAs in Table 3A

\begin{tabular}{ccccccc}
\hline \multicolumn{2}{c}{ Noise Present (Left) } & & & \multicolumn{2}{c}{ Noise Present (Right) } \\
\cline { 1 - 1 } \cline { 5 - 7 } Stimulus Type & Left & Right & & Stimulus Type & Left & Right \\
\hline S7 & NL & & & S3 & L & \\
S4 & & L & & S8 & & NL \\
S5 & N & L & & S4 & & L \\
S3 & L & & S6 & L & N \\
\hline
\end{tabular}

Note-In the interaction in the left ANOVA shown in Table 3A, the ERPs to left-sided spatially congruent bimodal stimuli (S7: NL left) and right-sided light stimuli (S4) are summed and compared with the sum of the ERPs to spatially incongruent stimuli (S5: $\mathrm{N}$ left and L right) and left-sided light stimuli (S3). Thus, in $(\mathrm{S} 7+\mathrm{S} 4)-(\mathrm{S} 5+\mathrm{S} 3)$, the physical stimulation is completely eliminated, and the influence of spatial congruency is isolated for a left-sided spatially congruent stimulus. The right part of the table illustrates the spatial congruency comparison for a right-sided spatially congruent stimulus: $(\mathrm{S} 8+\mathrm{S} 3)-(\mathrm{S} 6+\mathrm{S} 4)$. 
Table 4

Mean Reaction Time (RT, in Milliseconds) and Omission Rate (Omi) per Condition

\begin{tabular}{|c|c|c|c|c|c|c|c|c|c|c|c|}
\hline \multicolumn{6}{|c|}{ Targets (T1-T8) } & \multicolumn{6}{|c|}{ Combinations $(\mathrm{C} 1-\mathrm{C} 8)$} \\
\hline \multirow[b]{2}{*}{ Left } & \multirow[b]{2}{*}{ Right } & \multicolumn{2}{|c|}{ RT } & \multicolumn{2}{|c|}{ Omi } & \multirow[b]{2}{*}{ Left } & \multirow[b]{2}{*}{ Right } & \multicolumn{2}{|c|}{ RT } & \multicolumn{2}{|c|}{ Omi } \\
\hline & & $M$ & $S E$ & $M$ & $S E$ & & & $M$ & $S E$ & $M$ & $S E$ \\
\hline \multirow[t]{2}{*}{$\mathrm{NN}$} & & 566 & 17 & 4.5 & 1.2 & $\mathrm{NN}$ & $\mathrm{L}$ & 527 & 18 & 2.7 & 0.7 \\
\hline & NN & 564 & 16 & 2.4 & 0.7 & $\mathrm{~L}$ & NN & 542 & 17 & 2.2 & 0.6 \\
\hline \multirow[t]{2}{*}{ LL } & & 544 & 17 & 5.0 & 1.3 & LL & $\mathrm{N}$ & 539 & 20 & 4.9 & 2.0 \\
\hline & LL & 536 & 15 & 4.2 & 1.3 & $\mathrm{~N}$ & LL & 529 & 16 & 5.2 & 2.0 \\
\hline $\mathrm{NN}$ & LL & 479 & 15 & 1.5 & 0.5 & NNL & & 531 & 19 & 3.0 & 1.0 \\
\hline LL & NN & 486 & 16 & 1.5 & 0.4 & & NNL & 517 & 18 & 2.9 & 0.8 \\
\hline \multirow{2}{*}{ NNLL } & & 479 & 18 & 1.9 & 0.7 & LLN & & 538 & 18 & 7.9 & 3.0 \\
\hline & NNLL & 475 & 16 & 0.5 & 0.3 & & LLN & 532 & 17 & 6.4 & 1.6 \\
\hline
\end{tabular}

between 112 and $122 \mathrm{msec}$ and between 136 and $172 \mathrm{msec}$ after stimulus onset, indicating that the ERP to the bimodal stimulus was influenced by the position of the auditory stimulus component. Correspondingly, the light position $\times$ channel interaction was significant between
80 and $120 \mathrm{msec}$ and between 144 and $184 \mathrm{msec}$ after stimulus onset, indicating that the bimodal ERP was highly influenced by the position of the visual stimulus component. A spatial congruency effect, as revealed by a significant noise $\times$ light $\times$ channel interaction, was
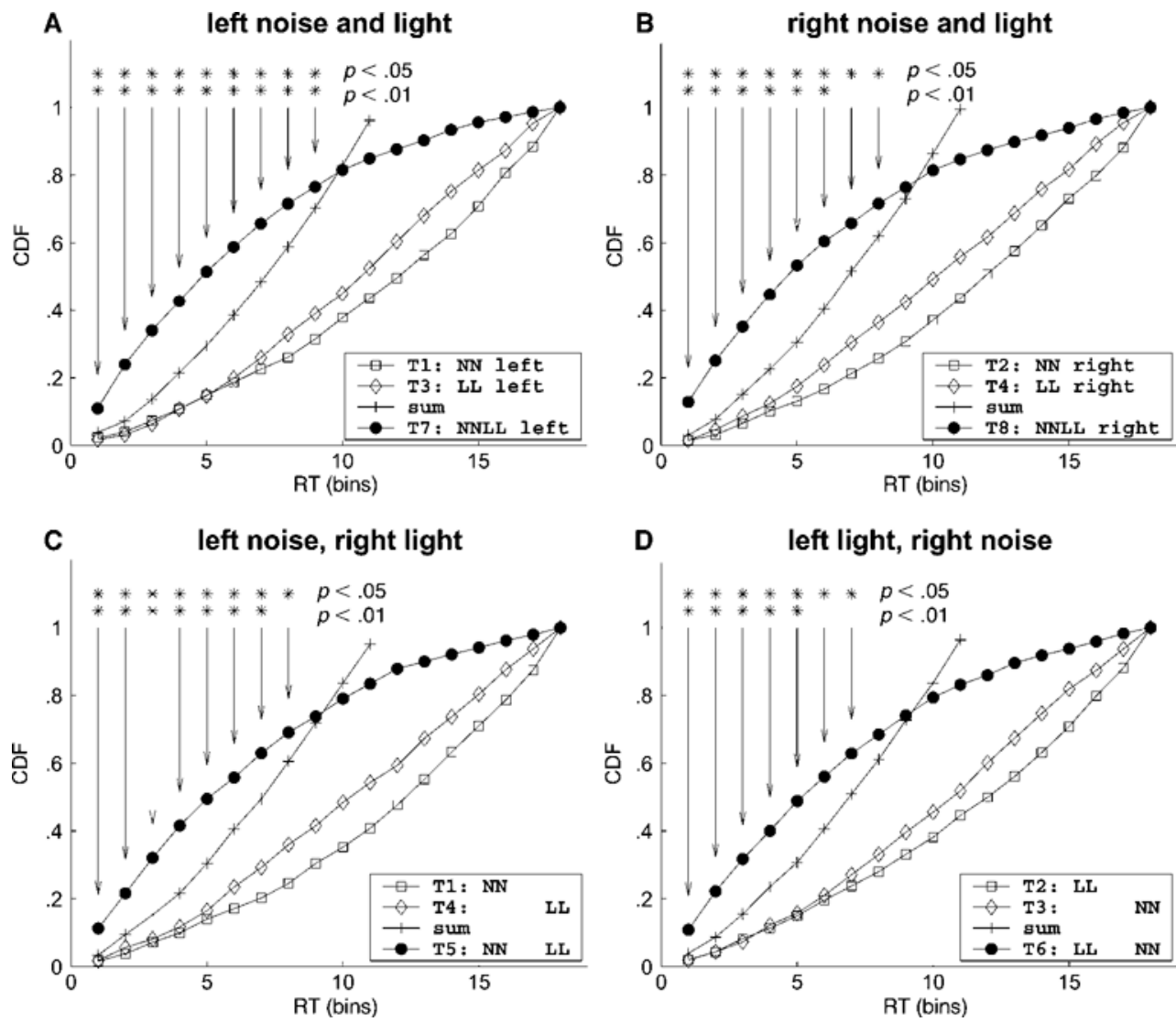

Figure 3. Observed cumulative reaction time (RT) distribution functions (CDFs) in the four redundanttarget conditions (T5-T8; see Table 1) and race model predictions from the respective RT CDFs to unimodal target stimuli (T1-T4). The upper two panels (A and B) show the conditions with spatially congruent redundant targets; the lower two panels (C and $D)$ show the conditions with spatially incongruent redundant targets. As can be seen in the lower ranges of all the figures, the proportions of fast responses to bimodal targets (filled circles) were higher than the summed $(+)$ respective proportions for unimodal targets (diamonds and squares). The stars indicate where this difference is significant (binomial tests as described by Corballis, 2002). 

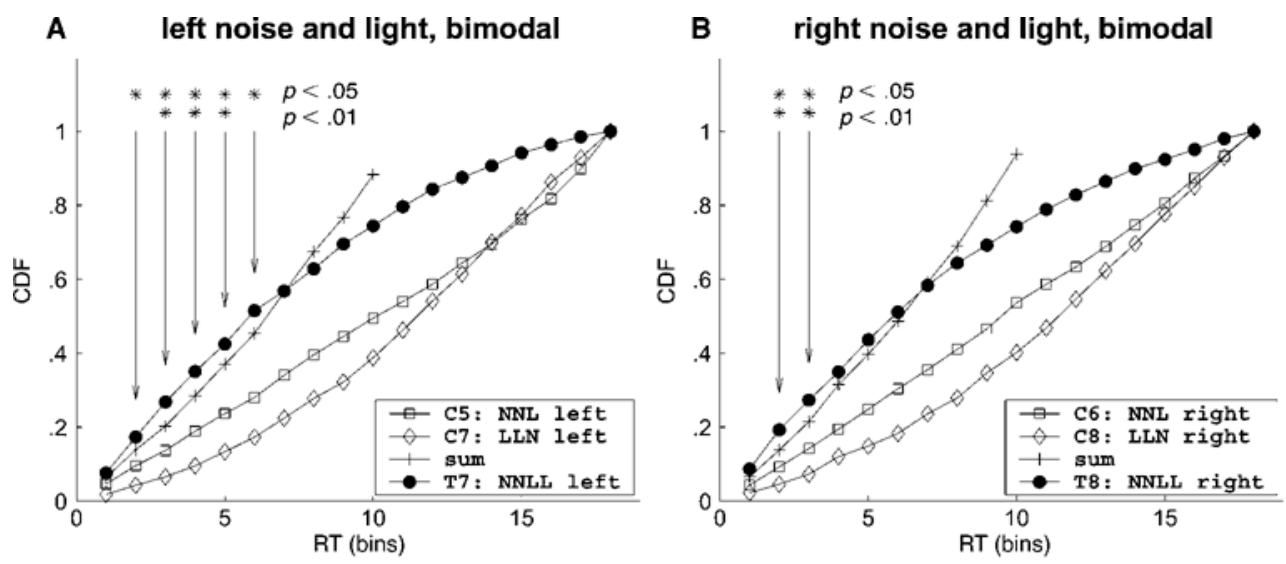

C left noise, right light, bimodal
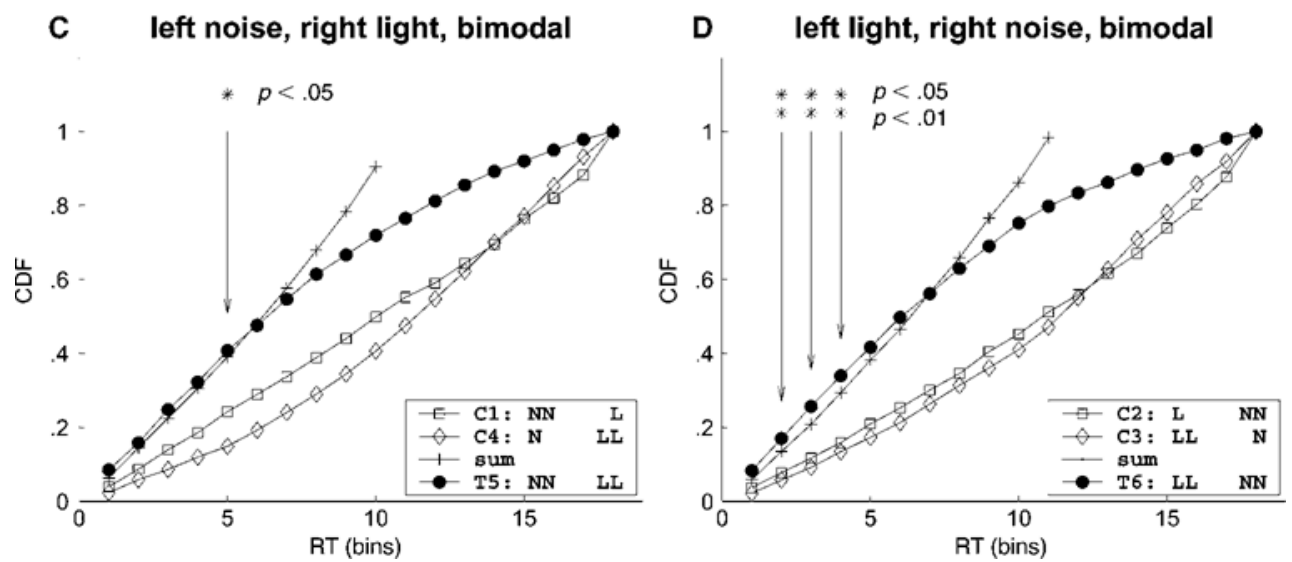

Figure 4. A modified race model test using only bimodal stimuli. Bimodal redundant targets are compared with the corresponding bimodal combinations of a standard and a target. In this modified race model test, the number of stimuli and the spatial relationship is kept constant, thereby isolating the response relevance. This enables a check for coactivation at later, decisional and executive processing stages. The amount of coactivation is lower than that in Figure 3. In panel $\mathrm{C}$, it fails to reach significance.

found between 150 and $166 \mathrm{msec}$ after stimulus presentation (see Figures 5-7). This effect indicates that the spatially congruent bimodal stimuli elicited different ERP topographies than were elicited when the two com- ponents of the bimodal stimulus were presented from different locations. Within this interaction, only the parietal contrast (comparing P3/P4 with their surroundings; see Figure 2) reached significance (at 154, 158, and

Table 5

ANOVA Results for Stimulus Laterality and the Spatial Congruency Effect

\begin{tabular}{|c|c|c|c|c|c|c|c|c|c|c|}
\hline \multirow{2}{*}{$\begin{array}{l}\text { Time } \\
(\mathrm{msec})\end{array}$} & \multicolumn{2}{|c|}{$\mathrm{L} \times \mathrm{C}$} & \multicolumn{2}{|c|}{$\mathrm{N} \times \mathrm{C}$} & \multicolumn{3}{|c|}{$\mathrm{L} \times \mathrm{N} \times \mathrm{C}$} & \multicolumn{3}{|c|}{$\mathrm{L} \times \mathrm{N} \times \mathrm{C}$ Contrasts } \\
\hline & $F$ & $p$ & $F$ & $p$ & $F$ & $p$ & eps & $F_{1}$ & $F_{2}$ & $F_{3}$ \\
\hline$\overline{130-140}$ & 0.70 & .56 & $2.87^{*}$ & .03 & 1.09 & .37 & .26 & .00 & 1.2 & 0.03 \\
\hline $140-150$ & 2.74 & .06 & $3.28^{*}$ & .02 & 2.61 & .07 & .20 & .05 & 2.40 & 0.77 \\
\hline $150-160$ & $5.93^{*}$ & $<.01$ & $3.66^{*}$ & $<.01$ & $3.78^{*}$ & .02 & .20 & 2.0 & 4.5 & 1.9 \\
\hline $160-170$ & $7.31^{*}$ & $<.01$ & $3.48^{*}$ & .02 & $3.59^{*}$ & .01 & .23 & 2.7 & $7.6^{*}$ & 2.6 \\
\hline $170-180$ & $5.42^{*}$ & $<.01$ & 2.75 & .06 & 1.94 & .12 & .27 & 2.9 & $11.0^{*}$ & 2.4 \\
\hline $180-190$ & $3.40^{*}$ & .03 & 1.47 & .24 & 0.76 & .56 & .27 & 2.6 & $9.5^{*}$ & 0.4 \\
\hline $190-200$ & 2.09 & .12 & 0.66 & .58 & 1.10 & .37 & .25 & 1.6 & 4.0 & 0.04 \\
\hline
\end{tabular}

Note-ANOVA results for the analysis outlined in Table 2A, for mean amplitudes of 10-msec time epochs. The interaction of light position and channel $(\mathrm{L} \times \mathrm{C})$ indicates different ERP topographies for left- and rightsided visual stimuli, and noise position $\times$ channel $(\mathrm{N} \times \mathrm{C})$ indicates different $\mathrm{ERP}$ topographies for left- and right-sided auditory stimuli, respectively. The three-way interaction $\mathrm{L} \times \mathrm{N} \times \mathrm{C}$ reflects differential processing of spatially congruent and spatially incongruent bimodal stimuli (see Table $2 \mathrm{~B}$ ). The three a priori contrasts $\left(F_{1}, F_{2}\right.$, and $\left.F_{3}\right)$ have been defined in Figure 2. $F_{2}$ compares the parietal channels $\mathrm{P} 3$ and $\mathrm{P} 4$ with the surroundings. $d f=16,176$ (1,176 for the contrasts). eps, Huynh-Feldt epsilon. $\quad{ }^{*} p<.05$. 
$160 \mathrm{msec}$ and from 170 to $180 \mathrm{msec}$ after stimulus onset), indicating that the spatial congruency effect observed here arises mainly over parietal recording leads. A similar pattern was observed in the target stimuli (Figure 8).

\section{Laterality of the Spatial Congruency Effect}

In the comparison above, the two spatially congruent bimodal stimuli were compared with the two stimuli with the auditory and visual components presented from different locations. Therefore, the results are not specific with respect to laterality. In order to examine whether the laterality of this effect depended on the side to which the spatially congruent stimulus was presented, modified analyses as outlined in Tables 3A and 3B were conducted.

In the first analysis, the ERPs to an unimodal visual stimulus on the left side and to a spatially congruent bimodal stimulus on the right side were summed ( $\mathrm{L}$ left + NL right). The resulting term was compared with the summed ERPs to a spatially incongruent stimulus and to a unimodal visual stimulus on the right side (L left and $\mathrm{N}$ right $+\mathrm{L}$ right). Mean amplitudes, $F$ values, and $p$ values of 10-msec time epochs are shown in Table 6 (right column). A spatial congruency effect, as revealed by a significant noise $\times$ light $\times$ channel interaction, emerged between 134 and $148 \mathrm{msec}$ after stimulus onset. The same analysis was run with the opposite laterality. For the spatially congruent stimulus on the left side, the comparison was significant between 132 and $144 \mathrm{msec}$ (Table 6, left column).

In a second analysis, the ERPs to a unimodal auditory stimulus on the left side and to a spatially congruent bimodal stimulus on the right side were summed ( $\mathrm{N}$ left + NL right). The resulting term was compared with the summed ERPs to a spatially incongruent stimulus and to a unimodal auditory stimulus on the right side ( $\mathrm{N}$ left and $\mathrm{L}$ right $+\mathrm{N}$ right). Mean amplitudes, $F$ values, and $p$ values of 10-msec time epochs are shown in Table 7 (right column). A spatial congruency effect, as revealed by a significant noise $\times$ light $\times$ channel interaction, was significant only for a short interval $(152-156 \mathrm{msec})$. The same analysis was run with opposite laterality. For the spatially congruent stimulus on the left side, the comparison did not reach significance; only a tendency toward higher $F$ values was found around $150 \mathrm{msec}$ (Table 7, left column).
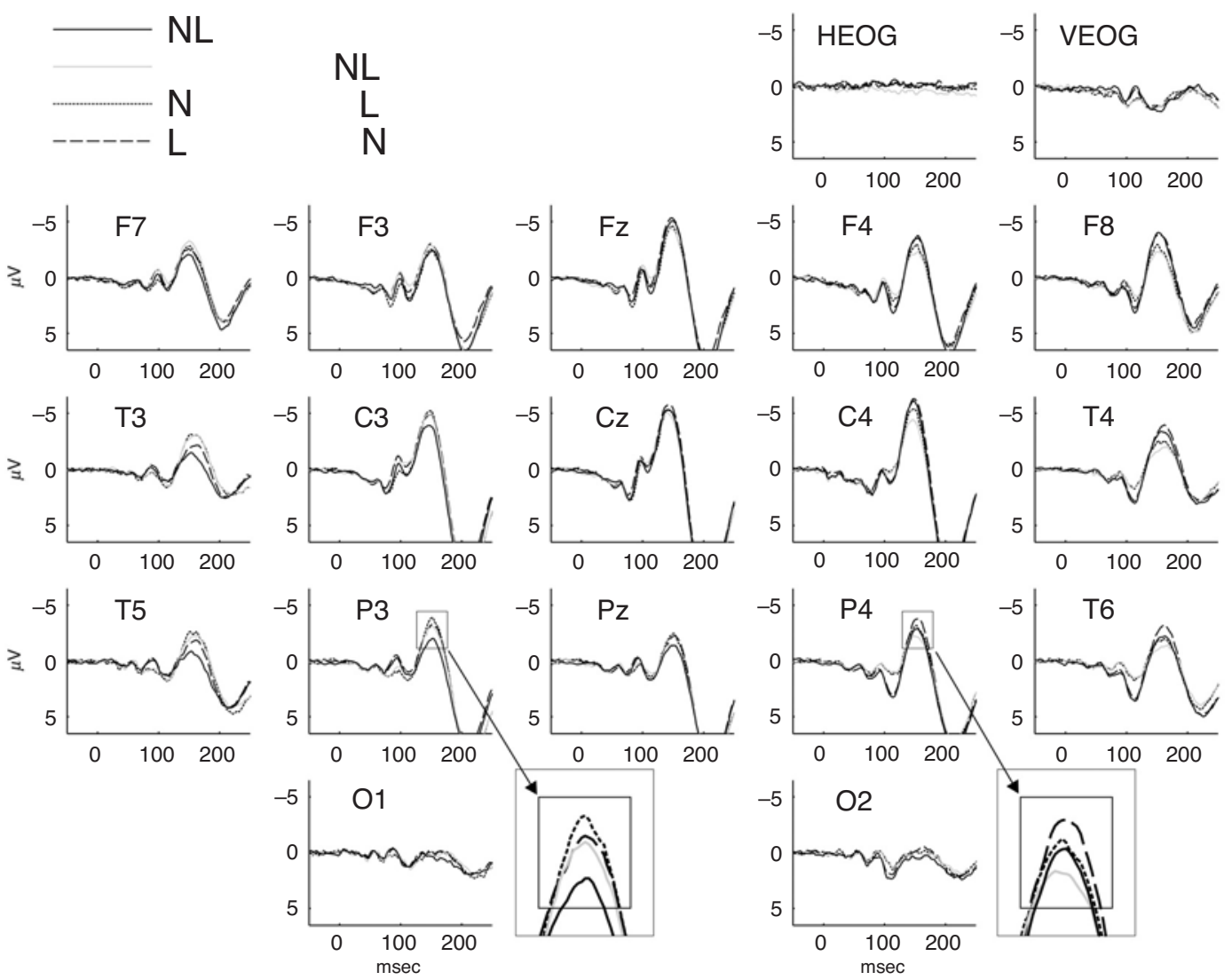

Figure 5. ERPs to the four bimodal standard conditions (see Table 1, left column). The comparison of the ERPs to spatially congruent and to spatially incongruent bimodal stimuli reveals a positive deflection over parietal recording leads, between 140 and 180 msec after stimulus onset. The difference is shown in Figure 6 (solid black line). N, noise; L, light. 

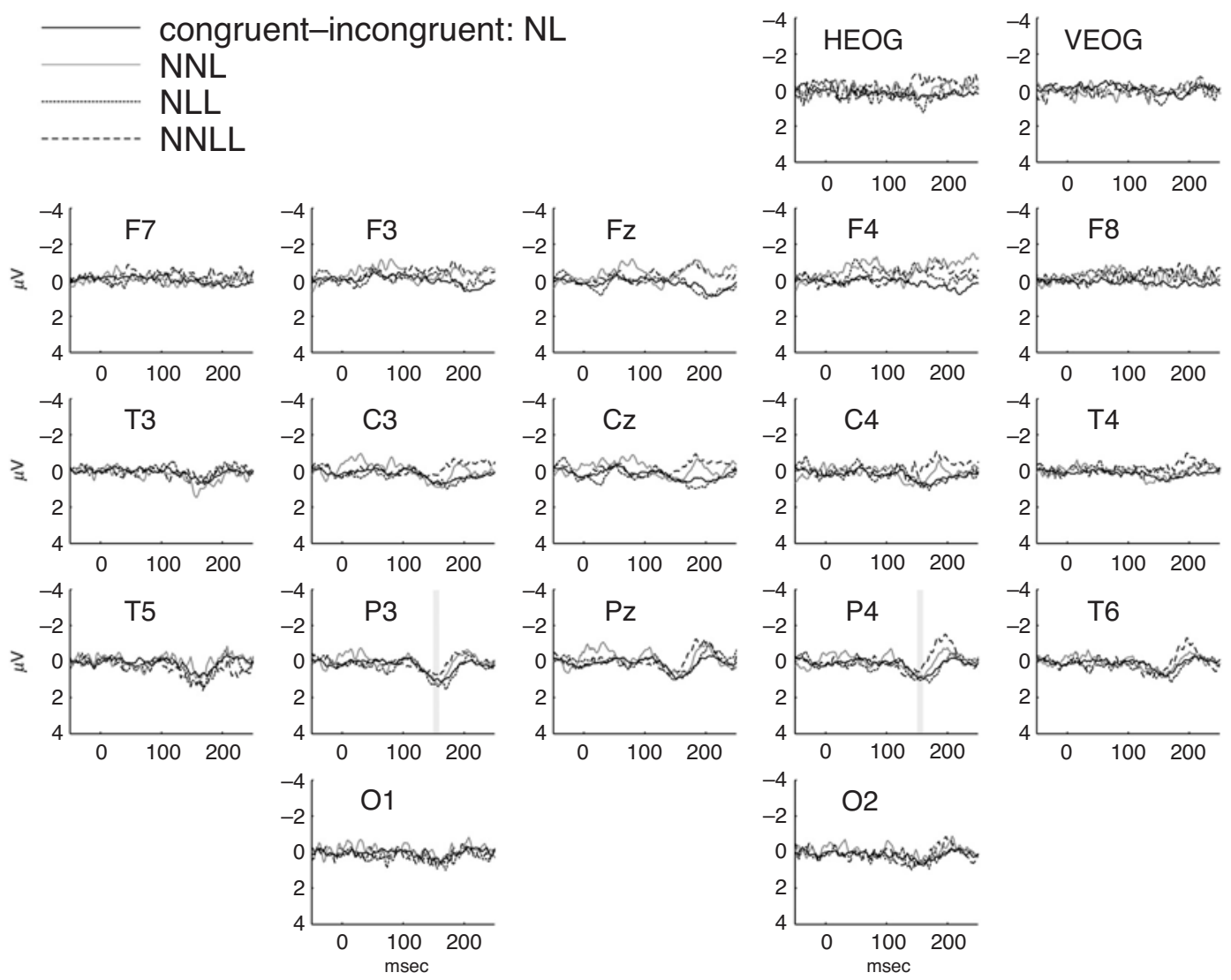

\begin{abstract}
Figure 6. Spatial congruency effect as obtained by the comparison of spatially congruent and spatially incongruent bimodal stimuli. The difference $(\mathbf{S 7}+\mathbf{S 8})-(\mathrm{S5}+\mathrm{S6})$ is shown in black. The same comparison was performed with bimodal targets (T7 + T8) - (T5 + T6), shown in dashed gray (NNLL), with combinations of noise standards and light targets $(\mathrm{C} 5+\mathrm{C6})-(\mathrm{C} 1+\mathrm{C2})$, shown in dotted gray (NLL), and with combinations of noise targets and light standards $(\mathrm{C} 7+\mathrm{C8})-(\mathrm{C} 3+\mathrm{C} 4)$, shown in solid gray $(\mathrm{NNL})$. In all four response relevance pairings, a spatial congruency effect emerges over parietal recording leads, around $150 \mathrm{msec}$ after stimulus onset (shown in P3 and P4). In the target conditions (NNLL, NLL, and NNL), the signal-to-noise ratio is lower because the condition was replicated only 80 times (standards: 430 times).
\end{abstract}

As Figure 8 shows, this laterality-specific spatial congruency effect had a parietal topography, contralateral to the spatially congruent stimulus.

\section{DISCUSSION}

In the present study, the spatial relationship of the two subcomponents of a bimodal stimulus was manipulated. Detection of bimodal stimuli was generally faster, and the RT gain in bimodal redundant targets was larger than that predicted by the race model. Responses for spatially congruent stimuli were faster than those for spatially incongruent stimuli, both for redundant targets and for bimodal combinations of standards and targets. ERPs revealed a spatial congruency effect starting at $150 \mathrm{msec}$ after stimulus onset, which had a parietal topography.

The RTE has been suggested to result from the activity of multisensory brain regions (e.g., Molholm et al., 2002; Schröger \& Widmann, 1998). Studies with single- cell recordings in the SC of the cat (Stein, 1998; Wallace et al., 1992) have shown that the temporal and the spatial relationships of the two components of a bimodal stimulus are crucial for multisensory interactions to occur. Hence, in the present study, the spatial relationship of the two stimulus components was manipulated by presenting spatially congruent (noise bursts and light flashes from the same position) and spatially incongruent (one subcomponent from the left position, one subcomponent from the right position) bimodal stimuli. If the RTE was a direct consequence of multisensory integration and if this integration was predominantly dependent on spatial congruency, an RT benefit should be expected only for spatially congruent redundant targets. That is, no RTE, or only race model effects, should occur for spatially incongruent bimodal stimuli.

Although a significant portion of the RTE gain could be explained by the spatial alignment, a race model violation was found for spatially incongruent bimodal stim- 

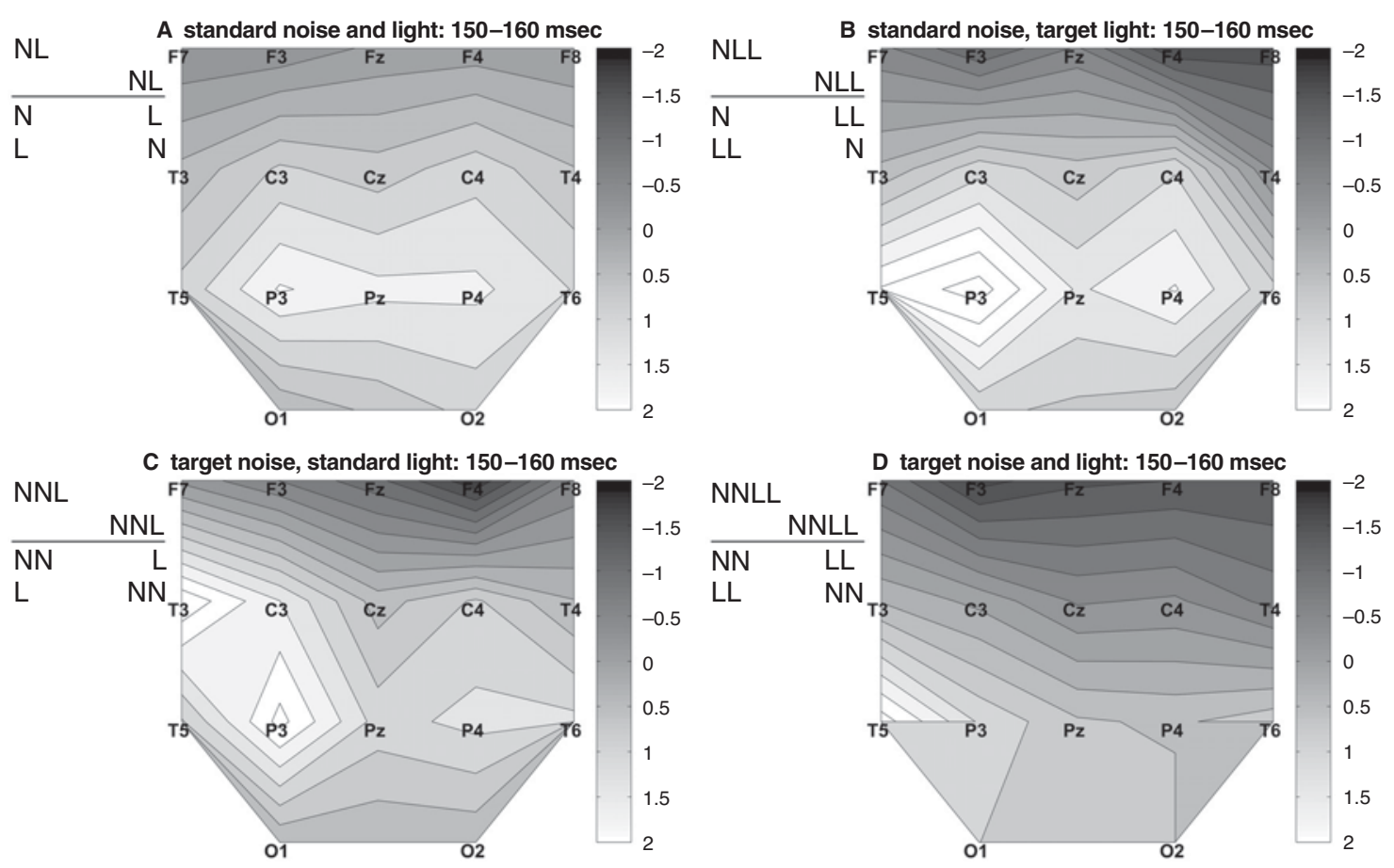

Figure 7. Spatial congruency effect, as obtained by the comparison of spatially congruent and incongruent bimodal stimuli (see Table 2B, Interaction column). The topographies show the interval of 150-160 msec in Table 5 (Figure 6) for bimodal standard stimuli. In addition, the effect is shown for standard-target combinations and redundant targets. $N$, NN, standard and target noise, L, LL, standard and target light.
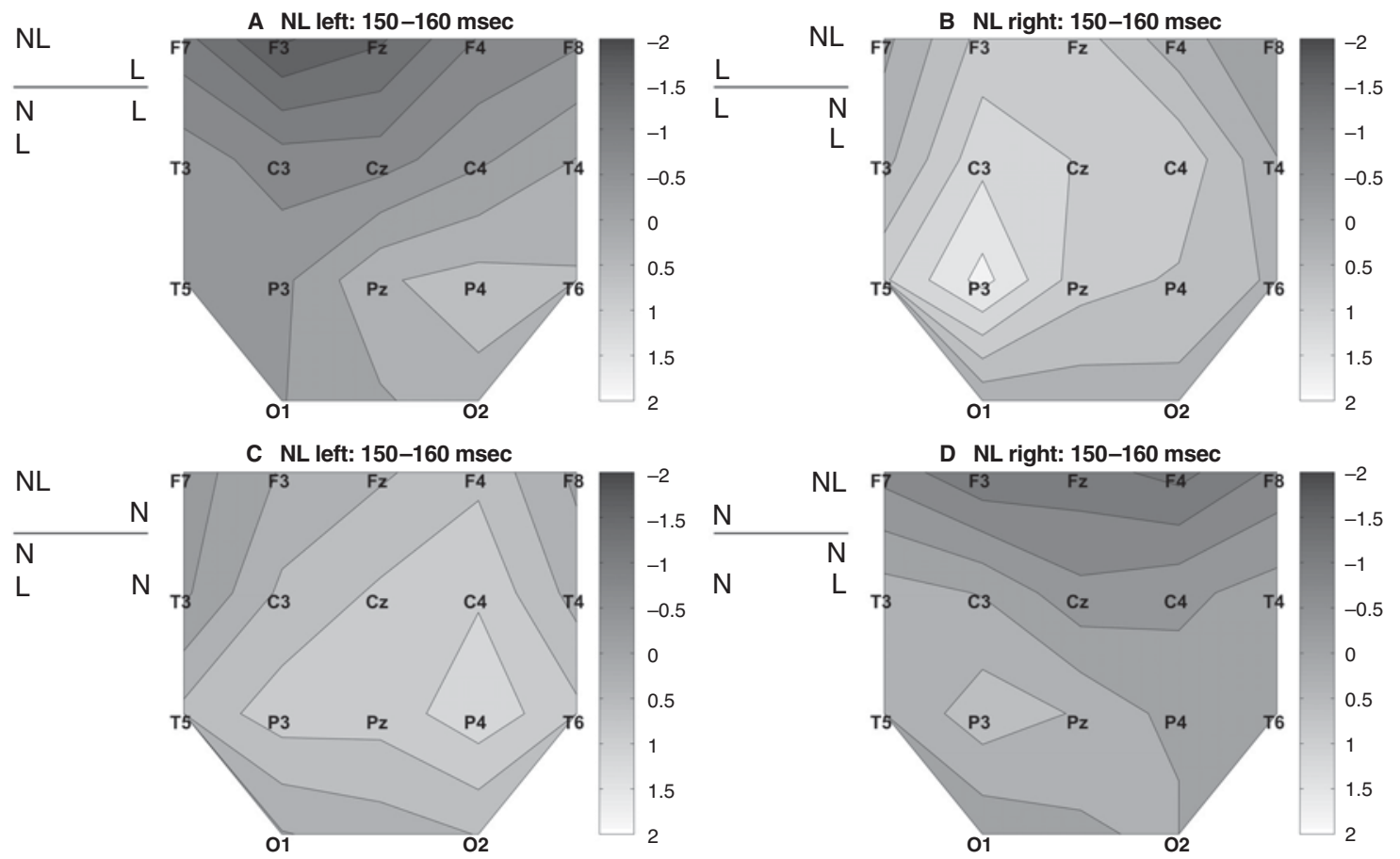

Figure 8. Laterality of the spatial congruency effect. The topographies show the interval of 150-160 msec in Tables 6 and 7 for spatially congruent bimodal stimuli at the specified location. The ERP difference for each topography is shown in the figure. As can be seen, the ERP deflection due to the spatial manipulation has a contralateral distribution with respect to the spatially congruent stimulus. This contralateral focus is independent of the laterality of the spatially incongruent stimulus ( $A, N$ left/L right; $C, L$ left/N right). 
Table 6

Laterality-Specific Spatial Congruency Effects

\begin{tabular}{|c|c|c|c|c|}
\hline \multirow[b]{2}{*}{ Time $(\mathrm{msec})$} & \multicolumn{2}{|c|}{$\begin{array}{l}\text { Noise }(\text { Left or Absent }) \\
\times \text { Light } \times \text { Channel } \\
(\mathrm{S} 7+\mathrm{S} 4)-(\mathrm{S} 5+\mathrm{S} 3)\end{array}$} & \multicolumn{2}{|c|}{$\begin{array}{l}\text { Noise }(\text { Absent or Right }) \\
\times \text { Light } \times \text { Channel } \\
(\mathrm{S} 8+\mathrm{S} 3)-(\mathrm{S} 6+\mathrm{S} 4)\end{array}$} \\
\hline & $F$ & $p$ & $F$ & $p$ \\
\hline $110-120$ & 2.0 & .13 & 1.6 & .18 \\
\hline $120-130$ & 2.1 & .10 & 2.4 & .05 \\
\hline $130-140$ & $3.8^{*}$ & $<.01$ & $3.7^{*}$ & .01 \\
\hline $140-150$ & $4.0^{*}$ & .01 & $3.7^{*}$ & .01 \\
\hline $150-160$ & 2.6 & .09 & 2.1 & .11 \\
\hline $160-170$ & 1.9 & .15 & 1.3 & .30 \\
\hline $170-180$ & 1.2 & .32 & 0.8 & .45 \\
\hline $180-190$ & 0.7 & .59 & 0.7 & .53 \\
\hline $190-200$ & 0.3 & .87 & 1.7 & .12 \\
\hline
\end{tabular}

Note-ANOVA results for the analysis outlined in Table 3A. Only the light $\times$ noise presence $\times$ channel interaction (Table $3 B$ ) is shown, indicating different ERP topographies for spatially congruent and spatially incongruent bimodal stimuli around $140 \mathrm{msec}$ after stimulus onset. The stimuli that were compared in the interaction are outlined in the column heads. The ERP topography for the interval of 150-160 msec is shown in Figure 8 (upper two graphs). $d f=16,176$; epsilon around $.25 . \quad{ }^{*} p<.05$.

uli as well (Figure 3). As was put forward in the Method section, three possible mechanisms may be responsible for this coactivation effect: (1) a general speeding of RTs to bimodal stimuli, as compared with unimodal stimuli, due to more stimulus energy (Bernstein et al., 1969), (2) a redundancy gain due to the coactivation of response codes in redundant targets, and (3) more efficient processing of spatially congruent stimuli. The direct comparison of the RTs to single targets (T1-T4) and to standardtarget-combinations $(\mathrm{C} 1-\mathrm{C} 8)$ suggests that mechanism (1) caused the largest RT gain in the present study, even though the second stimulus did not require any response. Since this mechanism operates across sensory channels, it may be regarded as the main multisensory contribution to the RTE.

Within the bimodal stimuli, we found a clear advantage for redundant targets, as compared with bimodal combinations of standards and targets (Mechanism 2). This is in line with findings of earlier redundant-target experiments with target detection tasks (Miller, 1991; Schröger \& Widmann, 1998). A separate test of the contribution of this mechanism is shown in Figure 4. The results indicate a relatively small, although significant, violation of the race model in all the conditions. Therefore, the activation of the two target-response associations seems to elicit a specific redundancy gain, unrelated to Mechanisms 1 and 2. The coactivation effect within the bimodal stimuli (Mechanism 2) seems to contradict the predictions of the dimensional action model (Cohen \& Shoup, 1997; Feintuch \& Cohen, 2002). For independent perceptual dimensions, this model assumes that spatial attention is necessary for coactivation to occur. Since the locations of the loudspeakers were $20^{\circ}$ to the left and to the right and the stimuli were presented in random order, the participants could not focus their attention on the location of the stimulus. Nevertheless, the available atten- tion in this situation of distributed spatial attention may have been sufficient to elicit coactivation.

However, an interpretation of the coactivation effect in Figure 4 as a redundancy gain may raise problems, because, in the present context, the standard of a standardtarget combination may have signaled the participants to withhold their response and, therefore, have acted like a no-go target. This response incompatibility may be associated with costs for standard-target combinations, whereas the redundant targets are unaffected. The small coactivation effect in Figure 4 might be explained by this response incompatibility effect. We tried to minimize response competition effects by instructing the participants to react as quickly as possible when they had detected "any target stimulus" and to ignore the standards. Nevertheless, the coactivation effect in Figure 4 cannot unequivocally be related to redundancy gains beyond the basic energy effect (Bernstein et al., 1969), and the dimensional action model of Cohen and Shoup (1997) needs more testing in the multisensory domain.

Finally, within the bimodal stimuli, the responses to spatially congruent stimuli were faster than the conditions in which the auditory and the visual components were presented from different locations (Mechanism 3). This spatial congruency effect was observed in both the redundant targets and in the standard-target combinations and thus seems to operate independently of the response relevance of the stimuli. It may, therefore, be concluded that the stage of processing at which the integration of the spatially congruent information occurs is not influenced by the response relevance of the stimulus components. A spatial congruency effect for bimodal stimuli has also been observed by Hughes et al. (1994) when participants had to selectively elicit a saccade to a visual stimulus that was accompanied by an auditory distractor. By contrast, for manual responses, Hughes et al. did not find a spatial congruency effect (however, only 3

Table 7

Laterality-Specific Spatial Congruency Effects (Replication)

\begin{tabular}{|c|c|c|c|c|}
\hline \multirow[b]{2}{*}{ Time (msec) } & \multicolumn{2}{|c|}{$\begin{array}{c}\text { Noise } \times \text { Light }(\text { Left or } \\
\text { Absent }) \times \text { Channel } \\
(\mathrm{S} 7+\mathrm{S} 2)-(\mathrm{S} 6+\mathrm{S} 1)\end{array}$} & \multicolumn{2}{|c|}{$\begin{array}{c}\text { Noise } \times \text { Light }(\text { Absent } \\
\text { or Right }) \times \text { Channel } \\
(\mathrm{S} 8+\mathrm{S} 1)-(\mathrm{S} 5+\mathrm{S} 2)\end{array}$} \\
\hline & $F$ & $p$ & $F$ & $p$ \\
\hline $110-120$ & 0.7 & .60 & 1.1 & .36 \\
\hline $120-130$ & 0.6 & .69 & 1.4 & .26 \\
\hline $130-140$ & 0.6 & .66 & 1.9 & .11 \\
\hline $140-150$ & 1.0 & .40 & 2.4 & .07 \\
\hline $150-160$ & 1.4 & .27 & 2.4 & .08 \\
\hline $160-170$ & 1.4 & .24 & 1.5 & .21 \\
\hline $170-180$ & 1.1 & .36 & 0.8 & .52 \\
\hline $180-190$ & 0.7 & .57 & 0.9 & .44 \\
\hline $190-200$ & 0.8 & .45 & 1.1 & .38 \\
\hline
\end{tabular}

Note-ANOVA results for the analysis outlined in Table 3A, using unimodal auditory stimuli together with the bimodal stimuli. The stimuli that were compared in the interaction are outlined in the column heads. The same results as those in Table 6 should be expected in the light presence $\times$ noise $\times$ channel interaction. The ERP topography for the interval of $150-160 \mathrm{msec}$ is shown in Figure 8 (lower two graphs). $d f=$ 16,176; epsilon around .25 . 
participants were investigated). Recently, Forster, CavinaPratesi, Aglioti, and Berlucchi (2002) ran a simple response RTE experiment with spatially congruent and spatially incongruent visuotactile stimuli but did not observe a spatial congruency effect. It may be argued that the low complexity of the simple response paradigm makes additional spatial effects unlikely (Posner, 1978). The spatial congruency effect observed in the present experiment has about the same size $(5 \mathrm{msec})$ as the interhemispheric transmission time (ITT; Poffenberger, 1912). Since the participants used the right foot to respond, the ITT can roughly be estimated as the difference between the RTs to left-sided stimuli minus the RTs to right-sided stimuli, which is about $7 \mathrm{msec}[F(1,11)=3.50, p<.05$, one-tailed]. It may, therefore, be hypothesized that the spatially congruent presentation of the bimodal stimuli did not elicit a specific spatial coactivation by itself but, rather, reflects the time needed to share the information of spatially incongruent stimuli between the two hemispheres. The ERP results may help to answer that question.

The comparison of ERPs for spatially congruent and spatially incongruent stimuli yielded a distributed ERP effect over the parietal leads that emerged between 140 and 180 msec. ${ }^{1}$ The latency of this difference wave is consistent with the findings of Fort et al. (2002), who observed a parieto-occipital positivity around $170 \mathrm{msec}$ in a subgroup of participants, and with Teder-Sälejärvi et al. (2002), who observed a central positivity around $175 \mathrm{msec}$. In these studies, the difference of the ERPs to bimodal and to the summed unimodal stimuli was evaluated. However, multisensory interactions were observed at both other electrode sites and other time points. The $\mathrm{AV}-(\mathrm{A}+\mathrm{V})$ difference consists of (1) the pure multisensory interaction, which may be location irrelevant, and (2) the effects of spatial congruency. The second aspect was isolated in the present study. If one agrees that the comparison of one versus two ERPs is problematic, in the sense that the activity revealed may not be related exclusively to multisensory interactions (Teder-Sälejärvi et al., 2002), the present data may be regarded as a valid subset of the possible neural interactions - namely, the spatial congruency portion (2). The early ERP effects, as observed, for example, in Molholm et al. (2002), would then be due to the pure multisensory interaction described in (1). In a single-cell study in macaques, Mazzoni et al. (1996) found multisensory neurons in the intraparietal area that responded very early to auditory $(30-250 \mathrm{msec}$, median at $150 \mathrm{msec})$ and visual $(60-210 \mathrm{msec}$, median at $150 \mathrm{msec}$ ) stimuli; hence, very early multisensory interactions cannot be ruled out but have to be confirmed with ERP studies avoiding the problematic $\mathrm{AV}-(\mathrm{A}+\mathrm{V})$ comparison.

We have shown that the RT gain observed for spatially congruent stimuli was similar for redundant targets and standard-target combinations. Note that with the timing of the target stimuli (70-msec light flash, 60-msec gap, 70 -msec flash) in the present experiment, the participants were not able to detect the presence of a target earlier than $70 \mathrm{msec}$ after stimulus onset (even more likely after
$130 \mathrm{msec}$, with onset of the second noise/light). Because spatial congruency modulated the ERP already at a latency of about $140 \mathrm{msec}$, this effect seems to be mainly perceptual, unrelated to specific target detection processes.

The maximum of the ERP congruency effect was located contralateral with respect to the side of the spatially congruent stimulus (Figure 8). This is in line with findings of Macaluso and Driver (2001), who observed activity in the intraparietal sulcus contralateral to spatially congruent visual and tactile stimuli with fMRI. The present data, therefore, suggest that spatial congruency enhances stimulus processing at perceptual stages and possibly involves multisensory brain regions in the parietal cortex. Whether the ERP spatial congruency effect reflects neural summation or a mechanism that detects the spatial congruency or incongruency of the stimuli cannot be decided on the basis of Figure 7: It might reflect a positivity elicited by the spatially congruent stimuli, as well as a negativity elicited by the spatially incongruent stimuli. The modified comparison shown in Figure 8 indicates that the underlying mechanism has a focal representation, contralateral to the spatially congruent stimulus. This laterality is observed in both Figures $8 \mathrm{~A}$ and $8 \mathrm{C}$, although the underlying ERP differences use spatially incongruent stimuli with opposite laterality (Figure $8 \mathrm{~A}, \mathrm{~N}$ left/L right; Figure $8 \mathrm{C}$, L left/ $\mathrm{N}$ right; similar for $8 \mathrm{~B}$ and $8 \mathrm{D}$ ). The fact that the focus of the spatial congruency effect depends solely on the laterality of the spatially congruent stimulus supports the interpretation that it is a positivity, elicited by the spatially congruent stimulus.

In summary, the present findings suggest that at least three different aspects of the redundancy gain can be distinguished that have been confounded in many RTE experiments in which simple manual RTs to stimuli at a constant position have been measured. First, the major portion of the RT gain seems to be an energy effect due the presence of two stimuli of different modalities, rather than one single stimulus (Bernstein et al., 1969; Welch \& Warren, 1986). This multisensory effect is independent of response relevance and of the spatial relationship of the two stimuli. Second, postperceptual coactivation due to redundant response relevance is very limited, as compared with the energy effect. Moreover, this portion of the RT gain might be explained by response competition when one, but not the other, component of the bimodal stimuli was response relevant (Figure 4). Third, processing of spatially congruent stimuli is faster than processing of spatially incongruent stimuli. This effect was smaller than expected, and evidence for coactivation was observed for spatially incongruent stimuli as well. The behavioral spatial congruency advantage was accompanied by an ERP effect starting as early as $150 \mathrm{msec}$ after stimulus onset (Figure 8), which had a parietal scalp topography with a contralateral dominance with respect to the spatially congruent stimulus. Therefore, the present findings underscore both the importance of space for cross-modal binding and the involvement of parietal brain structures in matching spatial coordinates across modalities. 


\section{REFERENCES}

Bernstein, I. H., Clark, M. H., \& Edelstein, B. A. (1969). Effects of an auditory signal on visual reaction time. Journal of Experimental Psychology, 80, 567-569.

Carlson, S., Pertovaara, A., \& Tanila, H. (1987). Late effects of early binocular visual deprivation on the function of Brodmann's area 7 of monkeys. Developmental Brain Research, 33, 101-111.

Cohen, A., \& Shoup, R. (1997). Perceptual dimensional constraints on response selection processes. Cognitive Psychology, 32, 128-181.

Corballis, M. C. (1998). Interhemispheric neural summation in the absence of the corpus callosum. Brain, 121, 1795-1807.

Corballis, M. C. (2002). Hemispheric interactions in simple reaction time. Neuropsychologia, 40, 423-434.

Diederich, A., \& Colonius, H. (1987). Intersensory facilitation in the motor component: A reaction time analysis. Psychological Research, 49, 23-29.

Feintuch, U., \& Cohen, A. (2002). Visual attention and coactivation of response decisions for features from different dimensions. Psychological Science, 13, 361-369.

Forster, B., Cavina-Pratesi, C., Aglioti, S. M., \& Berlucchi, G. (2002). Redundant target effect and intersensory facilitation from visual-tactile interactions in simple reaction time. Experimental Brain Research, 143, 480-487.

Fort, A., Delpuech, C., Pernier, J., \& Giard, M.-H. (2002). Early auditory-visual interactions in human cortex during nonredundant target identification. Cognitive Brain Research, 14, 20-30.

Foxe, J. J., Morocz, I. A., Murray, M. M., Higgins, B. A., Javitt, D. C., \& SCHROEDER, C. E. (2000). Multisensory auditory-somatosensory interactions in early cortical processing revealed by high-density electrical mapping. Cognitive Brain Research, 10, 77-83.

Giard, M. H., \& Peronnet, F. (1999). Auditory-visual integration during multimodal object recognition in humans: A behavioral and electrophysiological study. Journal of Cognitive Neuroscience, 11, 473490.

GirAy, M., \& UlRICH, R. (1993). Motor coactivation revealed by response force in divided and focused attention. Journal of Experimental Psychology: Human Perception \& Performance, 19, 12781291.

Gondan, M., Lange, K., Rösler, F., \& Röder, B. (2004). The redundant target effect is affected by modality switch costs. Psychonomic Bulletin \& Review, 11, 307-313.

Harrington, L. K., \& Peck, C. K. (1998). Spatial disparity affects visual-auditory interactions in human sensorimotor processing. $E x$ perimental Brain Research, 122, 247-252.

Hershenson, M. (1962). Reaction time as a measure of intersensory facilitation. Journal of Experimental Psychology, 63, 289-293.

Hughes, H. C., Reuter-Lorenz, P. A., Nozawa, G., \& Fendrich, R. (1994). Visual-auditory interactions in sensorimotor processing: Saccades versus manual responses. Journal of Experimental Psychology: Human Perception \& Performance, 20, 131-153.

HuYNh, H., \& FELDT, L. S. (1976). Estimation of the Box correction for degrees of freedom from sample data in randomized block and splitplot designs. Journal of Educational Statistics, 1, 69-82.

Lütkenhöner, B., Lammertmann, C., Simões, C., \& Hari, R. (2002). Magnetoencephalographic correlates of audiotactile interaction. NeuroImage, 15, 509-522.

Macaluso, E., \& Driver, J. (2001). Spatial attention and crossmodal interactions between vision and touch. Neuropsychologia, 39, 13041316.

Mazzoni, P., Bracewell, R. M., Barash, S., \& Andersen, R. A. (1996). Spatially tuned auditory responses in area LIP of macaques performing delayed memory saccades to acoustic targets. Journal of Neurophysiology, 75, 1233-1242.

Meredith, M. A., \& Stein, B. E. (1987). Spatial factors determine the activity of multi-sensory neurons in the cat's superior colliculus. Brain Research, 420, 162-166.

Miller, J. (1982). Divided attention: Evidence for coactivation with redundant signals. Cognitive Psychology, 14, 247-279.
Miller, J. (1986). Timecourse of coactivation in bimodal divided attention. Perception \& Psychophysics, 40, 331-343.

Miller, J. (1991). Channel interaction and the redundant-target effect in bimodal divided attention. Journal of Experimental Psychology: Human Perception \& Performance, 17, 160-169.

Miller, J., Ulrich, R., \& LAmarRe, Y. (2001). Locus of the redundantsignals effect in bimodal divided attention: A neurophysiological analysis. Perception \& Psychophysics, 63, 555-562.

Miniussi, C., Girelli, M., \& Marzi, C. A. (1998). Neural site of the redundant target effect: Electrophysiological evidence. Journal of Cognitive Neuroscience, 10, 216-230.

Molholm, S., Ritter, W., Murray, M. M., Javitt, D. C., Schroeder, C. E., \& Foxe, J. J. (2002). Multisensory auditory-visual interactions during early sensory processing in humans: A high-density electrical mapping study. Cognitive Brain Research, 14, 115-128.

Mordkoff, J. T., Miller, J., \& Roch, A.-C. (1996). Absence of coactivation in the motor component: Evidence from psychophysiological measures of target detection. Journal of Experimental Psychology: Human Perception \& Performance, 22, 25-41.

MoRdKoFf, J. T., \& YANTIS, S. (1991). An interactive race model of divided attention. Journal of Experimental Psychology: Human Perception \& Performance, 17, 520-538.

NiCKERSON, R. S. (1973). Intersensory facilitation of reaction time: Energy summation or preparation enhancement? Psychological Review, 80, 489-509.

Plat, F. M., Praamstra, P., \& Horstink, M. W. I. M. (2000). Redundantsignals effects on reaction time, response force, and movementrelated potentials in Parkinson's disease. Experimental Brain Research, 130, 533-539.

Poffenberger, A. T. (1912). Reaction time to retinal stimulation with special reference to the time lost in conduction through nerve centers. Archives of Psychology, 23, 1-73.

Posner, M. I. (1978). Chronometric exploration of mind. Hillsdale, NJ: Erlbaum.

RAAB, D. H. (1962). Statistical facilitation of simple reaction times. Transactions of the New York Academy of Sciences, 24, 574-590.

Reuter-Lorenz, P. A., Nozawa, G., Gazzaniga, M. S., \& Hughes, H. C. (1995). Fate of neglected targets: A chronometric analysis of redundant target effects in the bisected brain. Journal of Experimental Psychology: Human Perception \& Performance, 21, 221-230.

Roser, M., \& Corballis, M. C. (2002). Interhemispheric neural summation in the split brain with symmetrical and asymmetrical displays. Neuropsychologia, 40, 1300-1312.

Schröger, E., \& Widmann, A. (1998). Speeded responses to audiovisual changes result from bimodal integration. Psychophysiology, 35, 755-759.

SteIN, B. E. (1998). Neural mechanisms for synthesizing sensory information and producing adaptive behaviors. Experimental Brain Research, 123, 124-135.

Stein, B. E., \& Meredith, M. A. (1993). The merging of the senses. Cambridge, MA: MIT Press.

Teder-Sälejärvi, W. A., McDonald, J. J., Di Russo, F. D., \& HillYARD, S. A. (2002). An analysis of audio-visual crossmodal integration by means of event-related potential (ERP) recordings. Cognitive Brain Research, 14, 106-114.

Wallace, M. T., Meredith, M. A., \& Stein, B. E. (1992). Integration of multiple sensory modalities in cat cortex. Experimental Brain Research, 91, 484-488.

Welch, R. B., \& WARREN, D. H. (1986). Intersensory interactions. In K. R. Boff, L. Kaufman, \& J. P. Thomas (Eds.), Handbook of perception and human performance (pp. 25/1-25/36). New York: Wiley.

\section{NOTE}

1. Similar findings have recently been presented by Steven Hillyard, International Multisensory Research Forum, Sitges, Spain, June 2004.

(Manuscript received March 17, 2003; revision accepted for publication September 8, 2004.) 